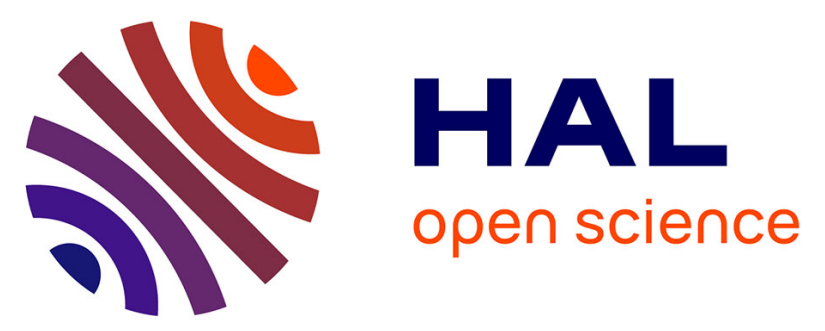

\title{
Scanning electron microscopy for quantitative small and large deformation measurements - part II: Experimental validation for magnifications from 200 to 10,000
}

\author{
M. A. Sutton, N. Li, David Garcia, Nicolas Cornille, Jean-José Orteu, S. R.
} Mcneill, H. W. Schreier, Xiaojian Li, A. P. Reynolds

\section{To cite this version:}

M. A. Sutton, N. Li, David Garcia, Nicolas Cornille, Jean-José Orteu, et al.. Scanning electron microscopy for quantitative small and large deformation measurements - part II: Experimental validation for magnifications from 200 to 10,000. Experimental Mechanics, 2007, 47 (6), p.789-804. 10.1007/s11340-007-9041-0 . hal-01644895

\section{HAL Id: hal-01644895 \\ https://hal.science/hal-01644895}

Submitted on 23 Feb 2018

HAL is a multi-disciplinary open access archive for the deposit and dissemination of scientific research documents, whether they are published or not. The documents may come from teaching and research institutions in France or abroad, or from public or private research centers.
L'archive ouverte pluridisciplinaire HAL, est destinée au dépôt et à la diffusion de documents scientifiques de niveau recherche, publiés ou non, émanant des établissements d'enseignement et de recherche français ou étrangers, des laboratoires publics ou privés. 


\title{
Scanning Electron Microscopy for Quantitative Small and Large Deformation Measurements Part II: Experimental Validation for Magnifications from 200 to 10,000
}

\author{
M.A. Sutton • N. Li • D. Garcia • N. Cornille • \\ J.J. Orteu • S.R. McNeill • H.W. Schreier • X. Li • \\ A.P. Reynolds
}

\begin{abstract}
A combination of drift distortion removal and spatial distortion removal are performed to correct Scan ning Electron Microscope (SEM) images at both $\times 200$ and $\times 10,000$ magnification. Using multiple, time spaced images and in plane rigid body motions to extract the relative displacement field throughout the imaging process, results from numerical simulations clearly demonstrate that the correction procedures successfully remove both drift and spatial distortions with errors on the order of \pm 0.02 pixels. A series of $2 \mathrm{D}$ translation and tensile loading experiments are performed in an SEM for magnifications at $\times 200$ and $\times 10,000$, where both the drift and spatial distortion removal methods described above are applied to correct the digital images and improve the accuracy of measurements obtained using 2D DIC. Results from translation and
\end{abstract}

M.A. Sutton $(\bowtie$, SEM member) • N. Li (SEM member) •

S.R. McNeill (SEM member) • X. Li (SEM member) ·

A.P. Reynolds

Department of Mechanical Engineering,

University of South Carolina,

Columbia, SC 29208, USA

e mail: Sutton@sc.edu

D. Garcia

108 Ennismore Common Lane,

Columbia, SC 29229, USA

H.W. Schreier (SEM member)

Correlated Solutions, Inc, 952 Sunset Boulevard,

West Columbia, SC 29252, USA

N. Cornille $\cdot$ J.J. Orteu

Ecole des Mines d'Albi, Albi, France loading experiments indicate that (a) the fully corrected displacement components have nearly random variability with standard deviation of 0.02 pixels $(\approx 25 \mathrm{~nm}$ at $\times 200$ and $\approx 0.5 \mathrm{~nm}$ at $\times 10,000$ ) in each displacement component and (b) the measured strain fields are unbiased and in excellent agreement with expected results, with a spatial resolution of 43 pixels $(\approx 54 \mu \mathrm{m}$ at $\times 200$ and $\approx 1.1 \mu \mathrm{m}$ at $\times 10,000)$ and a standard deviation on the order of $6 \times 10^{5}$ for each component.

Keywords Scanning electron microscopy $\cdot$ High and low magnification - Uniaxial tension experiment · Drift and distortion correction $\cdot 2 \mathrm{D}$ digital image correlation

\section{Introduction}

Accurate calibration of single camera $[1,2]$ or stereo vision systems at the micro scale, including the determination and correction of the underlying distortions in the measurement process, has received limited attention in the literature. One reason for this difficulty is the complexity of high magnification imaging systems, resulting in non parametric distortions that invalidate the common approaches for identifying and removing distortions in simple lens systems $[3,4]$.

In his pioneering work, Schreier et al. proposed a new methodology to calibrate accurately any imaging sensor by correcting a priori for spatial distortion using a non parametric model [5]. The correction process transforms the imaging sensor plane into a virtual distortion free sensor plane using simple translations of a speckled or gridded planar target. If a speckled target is translated, then the 
same target can be used to perform camera calibration of the distortion corrected virtual imaging sensor using un known arbitrary motions. As opposed to classical calibra tion techniques (relying on a dedicated target marked with fiducial points), this approach can be applied using any randomly textured planar object. This type of distortion depends only on the pixel position in the image and is designated as spatial distortion in this work.

Due to the nature of white light, optical imaging systems are limited to a maximum resolution that corresponds to a magnification of $\approx \times 1,000$. For higher spatial resolution imaging, systems based on electron microscopy (such as an SEM) have been used successfully. Since the physics of electron microscopy is quite different from optical micros copy, it became apparent early in the studies that a novel image analysis procedure, image model and calibration process would be necessary so that accurate measurements could be extracted from the digitized SEM images. For example, SEM systems have not only spatial distortion but also a temporally varying distortion, oftentimes known as drift distortion. In fact, most papers and even commercial SEM measurement systems simply ignore these effects and consider a pure projection model [6 11]. Though a few authors do take into account distortion (considering parametric distortion models [12 14]), the effect of drift distortion is generally not considered in experimental studies [15].

In a recent study, the authors proposed an imaging model and a distortion correction methodology to remove both drift and spatial distortions from SEM images [16], demonstrating that the method is effective for correcting SEM images at relatively low magnification.

In this study, "Scan Process Modeling in an SEM" and "Imaging Model for Planar Object" Sections presents additional details for the method described in [16]. "Numerical Simulations" Section presents numerical simu lation results confirming that the procedure for quantifying drift and spatial distortion is effective. "Experiments" Section overviews the pattern development, experimental setup and image acquisition procedures, presenting results from a series of 2D experiments using an SEM to obtain data at $\times 200$ and $\times 10,000$ magnifications. "Discussion" Section discusses the results and highlights key aspects of the measurement methodology. "Concluding Remarks" Section provides concluding remarks.

\section{Scan Process Modeling in an SEM}

As discussed in Part I, each SEM image is generated pixel by pixel following a rastering process. Each pixel requires a dwell time, $t_{D}$, to define the "intensity" of image at that location, so that the required scanning time for an entire row, $t_{R}$, and an entire frame, $t_{F}$, of image are given by

$\boldsymbol{t}_{R}=\boldsymbol{W} \boldsymbol{t}_{D}+\boldsymbol{t}_{j}$

$t_{F}=H t_{R}=H W t_{D}+H t_{J}$

where $W$ is the width of the image in pixels and $H$ is the height of image in pixels, $t_{J}$ is the time delay in seconds for repositioning the e beam and stabilizing it to the next row. ${ }^{1}$ Since the $(x, y)$ position in the image is in direct correspon dence with the scan time, one can write

$\boldsymbol{t}(\boldsymbol{x}, \boldsymbol{y})=\boldsymbol{x} \boldsymbol{t}_{D}+\boldsymbol{y} \boldsymbol{t}_{R}$

where $0 \leq x \leq W-1$ and $0 \leq y \leq H-1$. Thus, $t=0$ corresponds to the beginning of the scan for pixel $(0,0)$. Equation (3) implies that two pixel positions, and hence two times, will generally experience much different drift functions. Such differences have been observed for con secutive rows in an image, with a clearly defined shift in drift measured when moving from the last pixel in one row to the first pixel in the next row.

If one considers the time delay between acquiring consecutive images, then the total time from the beginning of the scan for the first image to the current time in the $n$th image for a given location $(x, y)$ is written

$T(x, y)=\sum_{n}^{N} t_{n}+(N-1) t_{F}+t(x, y)$

where $t_{n}$ is the recorded delay time between images.

\section{Imaging Model for Planar Object}

It is assumed that (a) the specimen is nearly planar, (b) translations and/or rotations occur within the plane of the specimen, and (c) deformations applied to the specimen occur within the plane of the specimen. Thus, the imaging process can be viewed as being a plane to plane transformation.

Assuming a perspective projection model for the imaging process, the equations in terms of undistorted coordinates in the image plane can be written in the simple form

$\left[\begin{array}{l}x \\ y\end{array}\right]=\left[\begin{array}{lr}M_{x} & 0 \\ 0 & M_{y}\end{array}\right]\left[\begin{array}{l}x_{0} \\ y_{0}\end{array}\right]+\left[\begin{array}{l}C_{x} \\ C_{y}\end{array}\right]$

where $(x, y)^{T}$ are in pixel coordinates; $\left(M_{x}, M_{y}\right)^{T}$ transform object coordinates $\left(x_{0}, y_{0}\right)^{T}$ into the sensor plane and have units pixel $/ \mathrm{mm}$ on the object and $\left(C_{x}, C_{y}\right)^{T}$ designates the location of the image center in the sensor plane. Additional "sensor plane scale factors" may be determined by

\footnotetext{
${ }^{1}$ For images integrated over M scans, the dwell time is the sum of the dwell times for each integrated scan.
} 


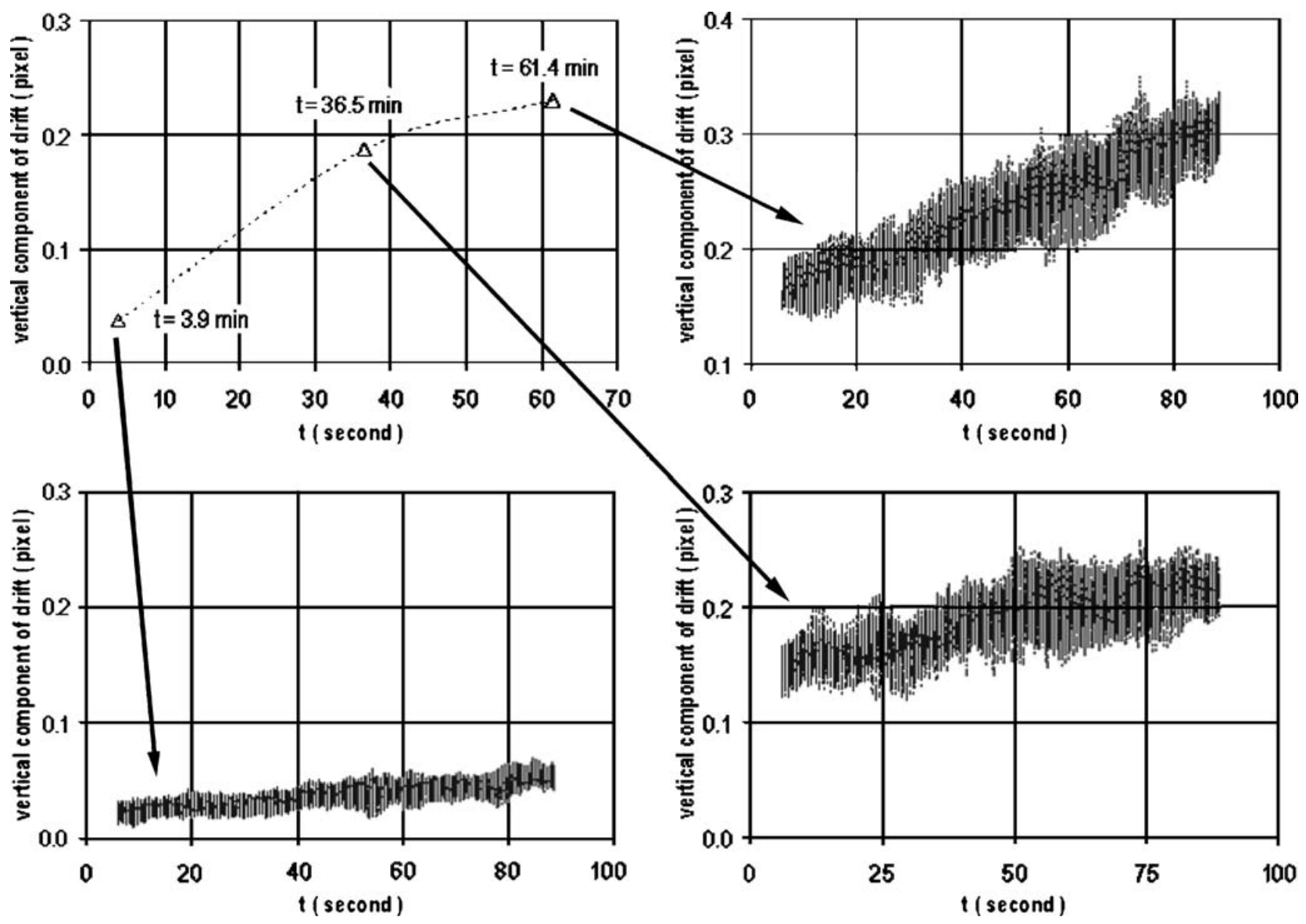

Fig. 1 Non uniform image drift in the vertical direction within each image at $\times 200$. Drift variations are primarily due to time representation of the data, where step changes occur at end of each line

manufacturer information regarding the physical size of the pixels that are used to digitize the image, though their values are not required unless the true magnification factor is desired. As shown in equation (5), there are four unknown parameters in the image model.

\section{Image Distortions in an SEM}

It is assumed that the distortions in an SEM can be repre sented by drift and spatial vector functions [16]. First, $\mathbf{D}_{\mathrm{dr}}(t)=\left[\delta_{x}(t), \delta_{y}(t)\right]^{T}$ is defined as the drift distortion function in two orthogonal directions, where $t$ is the scan time and $t \in[0, \infty)$. Second, $\mathbf{D}_{\mathrm{sp}}(\mathbf{r})=\mathbf{D}_{\mathrm{sp}}(x, y)$ is defined as the spatial distortion function in two orthogonal directions, where $\mathbf{r}=(x, y)^{T}$ is the undistorted pixel position of a point on the image plane. Thus, the distorted position $\mathbf{R}$ of the point can be written;

$\mathbf{R}=\mathbf{r}+\mathbf{D}_{\mathrm{dr}}(\boldsymbol{t})+\mathbf{D}_{\mathrm{sp}}(\mathbf{r})$

Since the distorted positions of points, $\mathbf{R}$, are the measurable quantities using DIC, the inverse functions for both drift and spatial distortion are the quantities obtained during the calibration process $^{2}$ so that the undistorted (ideal) positions of the points, $\mathbf{r}$, can be estimated.

\section{Drift Distortion}

Drift distortion, or temporally varying distortion, is present at all magnifications in an SEM, though it is particularly noticeable at high magnification. Figure 1 presents mea surements of the vertical drift displacement from the beginning to the end of a scan for images acquired at three different times. The data shows three trends that the authors have observed at all magnifications. First, there is random, pixel to pixel noise throughout the scan process. Second, drift displacement changes with time in a non linear manner. Third, the magnitude of the relative drift within each image changes with time. As shown in Fig. 1, the drift distortion within an image ranges up to 0.37 out of 1,024 pixels, introducing a strain error of $\approx 3.7 \times 10^{4}$.

To quantify the drift distortion at each pixel throughout an experiment, several approaches have been investigated.

\footnotetext{
${ }^{2}$ Though the focus of this study is on 2D image correlation (a single view), the procedure can be applied to each view in a stereo system to remove distortions.
} 
Preliminary experiments demonstrated that a global model cannot adequately represent experimental observations whereas a local model will provide good agreement with experimental measurements. Based on these investigations, a novel drift correction procedure has been developed [16] that is consistent with experimentally observed SEM imaging.

\section{Spatial Distortion}

In simple lens systems, e.g. a typical digital camera, spatially varying distortion (spatial distortion or image distortion) is a well known problem. The commonly used method for modeling such imaging systems assumes that the distortions (deviations from the ideal image positions) are due to factors such as lenses aberrations, misalignment of optical elements, non parallelism between image plane and sensor plane, lens curvature imperfection, each of which can be estimated using established parametric models.

\section{Parametric model}

Classical models used to estimate the spatial distortions are parametric in nature [17 19], typical forms include radial distortion, de centering distortion, prismatic distortion and tangential distortion. For example, one may write a radial distortion function, $\mathbf{F}$, in the form

$$
\begin{aligned}
& \left(x_{d}, y_{d}\right)^{T}=\mathbf{F}\left(r^{m} ; C_{x}, C_{y}\right) \\
& r^{2}=\left(x-C_{x}\right)^{2}+\left(y-C_{y}\right)^{2}
\end{aligned}
$$

where $\left(x_{\mathrm{d}}, y_{\mathrm{d}}\right)^{T}$ is the distorted position in the image plane of the undistorted point $(x, y)^{T}$ and $\mathrm{m}$ is the power of $\mathrm{r}$ to be a parameter used in the distortion model. Typically, the distortion function parameters are obtained at the same time as the imaging parameters in equation (5) using a non linear optimization process.

\section{Non parametric model}

If non parametric models for distortion correction are employed, then the role of the center in the mapping

\section{Calibration Phase}

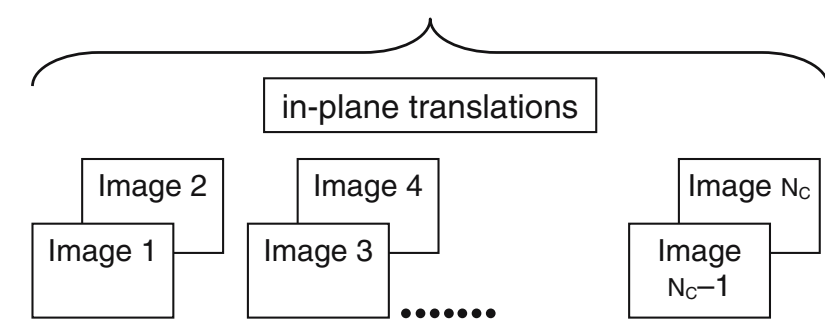

drift distortion estimation and spatial distortion determination process generally is embedded in the distortion correction process and is not determined separately. If distortion correction is performed prior to calibration, then the only parameters to be determined during the calibration process are the magnification factors. The magnification factors typically are determined through (a) known motions of the object, (b) points on the object with known spacing, or (c) combinations of both. If distortion correction is not performed prior to calibration, then the magnification factors and the distortion parameters typically are obtained through the calibration process.

Since the SEM imaging process is based upon the interaction between atoms of the observed specimen and an e beam, as well as scanning and focusing processes that employ electro magnetic principles to perform the required functions, pre specified classical distortions are unlikely to be effective when used to estimate arbitrary aberrations or unknown (but deterministic) distortions in a complex imaging system such as an SEM. To deal with this, the method outlined by Schreier et al. [5], which employs B Splines or other general forms, can be used to quantify the full field spatial distortions present in an SEM image.

Finally, it is noted that accurate grid targets are used in typical imaging systems to quantify spatial distortions. Since accurate grid targets used in previous non parametric distortion studies $[20,21]$ are likely to be difficult to realize at the micro or nano scale for the SEM, the method developed recently $[5,22]$ that employs arbitrary, unknown translations of the randomly speckled specimen for calibra tion at reduced length scales, is used in this current study.

\section{Drift and Spatial Distortion Correction for Calibration}

Figure 2 presents a schematic of the processes used to (a) quantify both drift and spatial distortion fields across the images, (b) calibrate the SEM, and (c) measure the desired deformations during an experiment. As shown on the left side of Fig. 2, pairs of images are acquired of the specimen as it is undergoing a series of in plane translations. Each pair of images is acquired without specimen motion. The

\section{Measurement Phase}

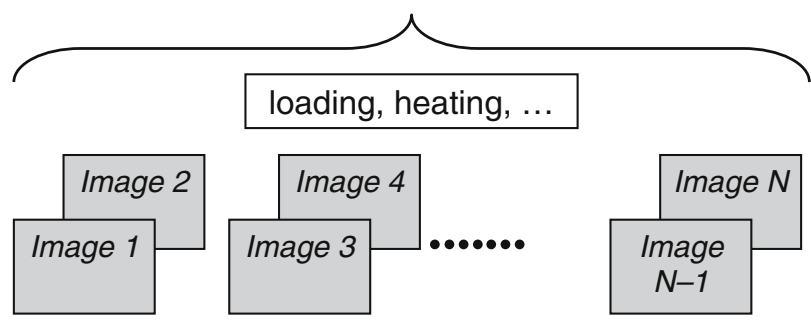

new drift distortion estimation with spatial distortion removal, deformation determination

Fig. 2 Schematic of overall image acquisition, image correction and deformation measurement process in the SEM 


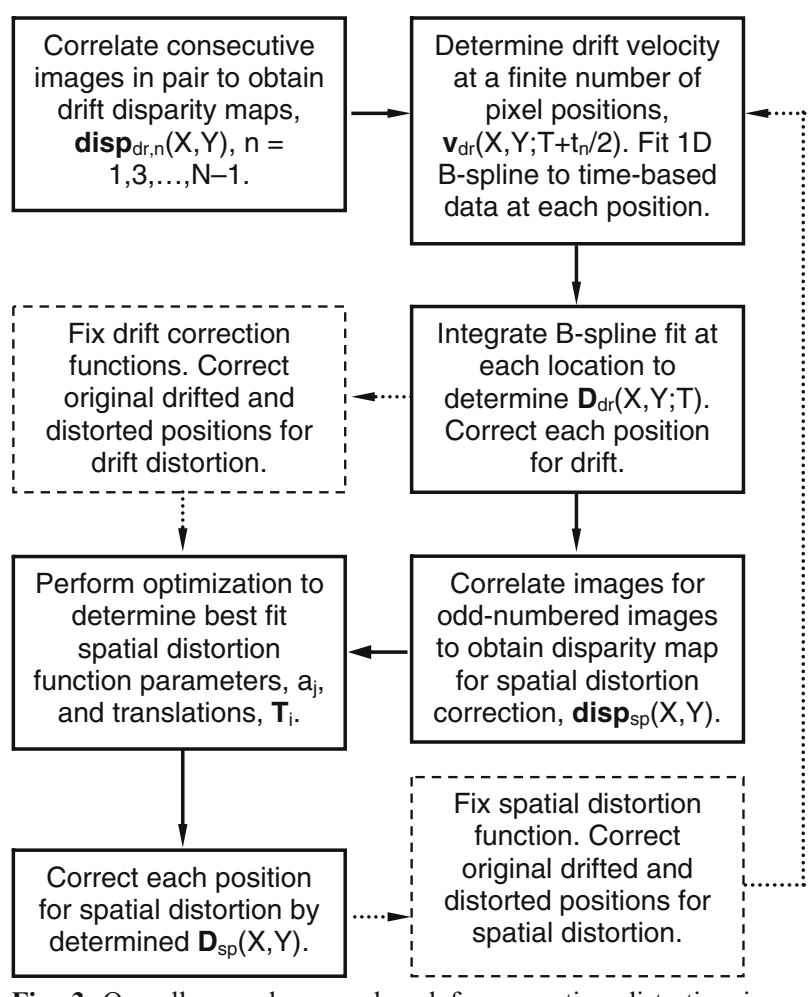

Fig. 3 Overall procedure employed for correcting distortion in an SEM. Relaxation methods are implemented during process to improve convergence

image pairs are used to estimate the drift distortion at each pixel location in the image. The translation sequence, which includes both horizontal and vertical motions, is used to extract the spatial distortion.

Figure 3 presents a summary of the procedures used to correct for drift and spatial distortion. To correct the drift distortion, 2D DIC is performed to determine the drift dis placement vector, $\operatorname{disp}_{\mathrm{dr}}(X, Y)$, for each fixed position $(X, Y)$ in the sensor plane. For a given image pair using the equation

$$
\begin{aligned}
& \operatorname{disp}_{\mathrm{dr}, n}(X, Y)=\mathbf{D}_{\mathrm{dr}}\left(T+t_{n}+t_{\mathrm{disp}}\right) \quad \mathbf{D}_{\mathrm{dr}}(T) \\
& t_{\mathrm{disp}}=\left[\delta_{x}\left(T+t_{n}\right) \quad \delta_{x}(T)\right] t_{D}+\left[\delta_{y}\left(T+t_{n}\right) \quad \delta_{y}(T)\right] t_{R}
\end{aligned}
$$

where $\left[\delta_{x}\left(t+T_{n}\right)-\delta_{x}(t), \delta_{y}\left(t+T_{n}\right)-\delta_{y}(t)\right]^{T}$ is the mea sured difference in the displacement vector at the pixel correspond to a given time, $T$, and time $T$ is given by equation (4). Using equation (8), the velocity of drift for each position, $v_{\mathrm{dr}, n}\left(X, Y ; T+t_{n} / 2\right)$, can be estimated using a central finite difference form. At each position $(X, Y)$, $\mathbf{v}_{\mathrm{dr}}(X, Y ; T)$ is fitted with a B Spline function in time and integrated over time to determine the drift for each position $(X, Y)$ in the $n$th odd numbered image, ${ }^{3} \mathbf{D}_{\mathrm{dr}, \mathrm{n}}(X, Y), n=1,3, \ldots$,

\footnotetext{
${ }^{3}$ For the initial image pair, variations in disparity across the image requires a piecewise integration over the disparity field between $(0,0)$ and $(X, Y)$, so that the drift at any position $(X, Y)$ can be determined. A less accurate approach would be to integrate the estimated $\operatorname{disp}_{\mathrm{dr}}(X, Y$; $T$ ) from $T 0$ to the time corresponding to $(X, Y)$.
}

$N$ 1. The drift vector, $\mathbf{D}_{\mathrm{dr}, \mathrm{n}}(X, Y)$, is used to correct the odd numbered images $n=1,3, \ldots, N 1$ for the drift distortion.

Once the odd numbered images are corrected for drift, they are used again for image correlation. Assuming a functional form for the disparity map over the region of interest (e.g., a 2D B Spline), the disparity maps (i.e., the difference in displacement for image locations that have undergone rigid body motion) are used in a least square procedure to determine both the rigid body motions and also the best fit B Spline parameters for the disparity data [5]. Typically, the best estimates for the rigid body motions and the B Spline fitting parameters are determined simultaneously.

Because SEM images and the corresponding disparity maps have considerable electronic and measurement noise (see Fig. 1 for graphical presentation and also the discussion in "Introduction" and "Scan Process Modeling in an SEM" Sections of Part I), ${ }^{4}$ the local drift correction model should employ a reduced order for the B Spline fit to the disparity vector data so that smoothing of the data is performed during the fitting process. This is particularly important for any differential integral approach since noisy data will introduce large oscillations in the derivatives and the resulting drift vector estimates will be less accurate.

\section{Numerical Simulations}

\section{Generation of Disparity Data}

Simulations are performed for both calibration phase and measurement phase $^{5}$ in a manner that is consistent with actual experimental conditions, with the positions of points estimated theoretically without use of the 2D DIC process $[24,25]$. Using pre defined functional forms the distorted positions of a finite number of locations, $(X, Y)_{k}$, are determined for each image $n$. The process is repeated several times, so that the distorted positions $(X, Y)_{k n}, k=$ $1,2, \ldots, \mathrm{K}, n=1,2, \ldots, N$, are determined. ${ }^{6}$

With $t_{n}=120 \mathrm{~s}, t_{D}=10^{4} \mathrm{~s}, t_{R}=1.071 \times 10^{2} \mathrm{~s}$ and an imaging array size of $1,024 \times 884$, the positions of image

\footnotetext{
${ }^{4}$ Sources of measurement error in an SEM may also include the effects of environmental factors such as mechanical vibrations and sound.

${ }^{5}$ The procedure whereby the drift distortion is computed separately for the calibration and measurement phases is used in practice to minimize the effects of specimen shifts during the initial loading process. However, in principle the process can be continuous.

${ }^{6}$ In practice, 7 to 11 pairs of images are acquired during the calibration phase; the only requirement is that several translations in two orthogonal directions be performed. The number of pairs of images during the measurement phase will vary with the number of strain increments; for better estimation of B Spline function it should be more than six pairs of images acquired.
} 


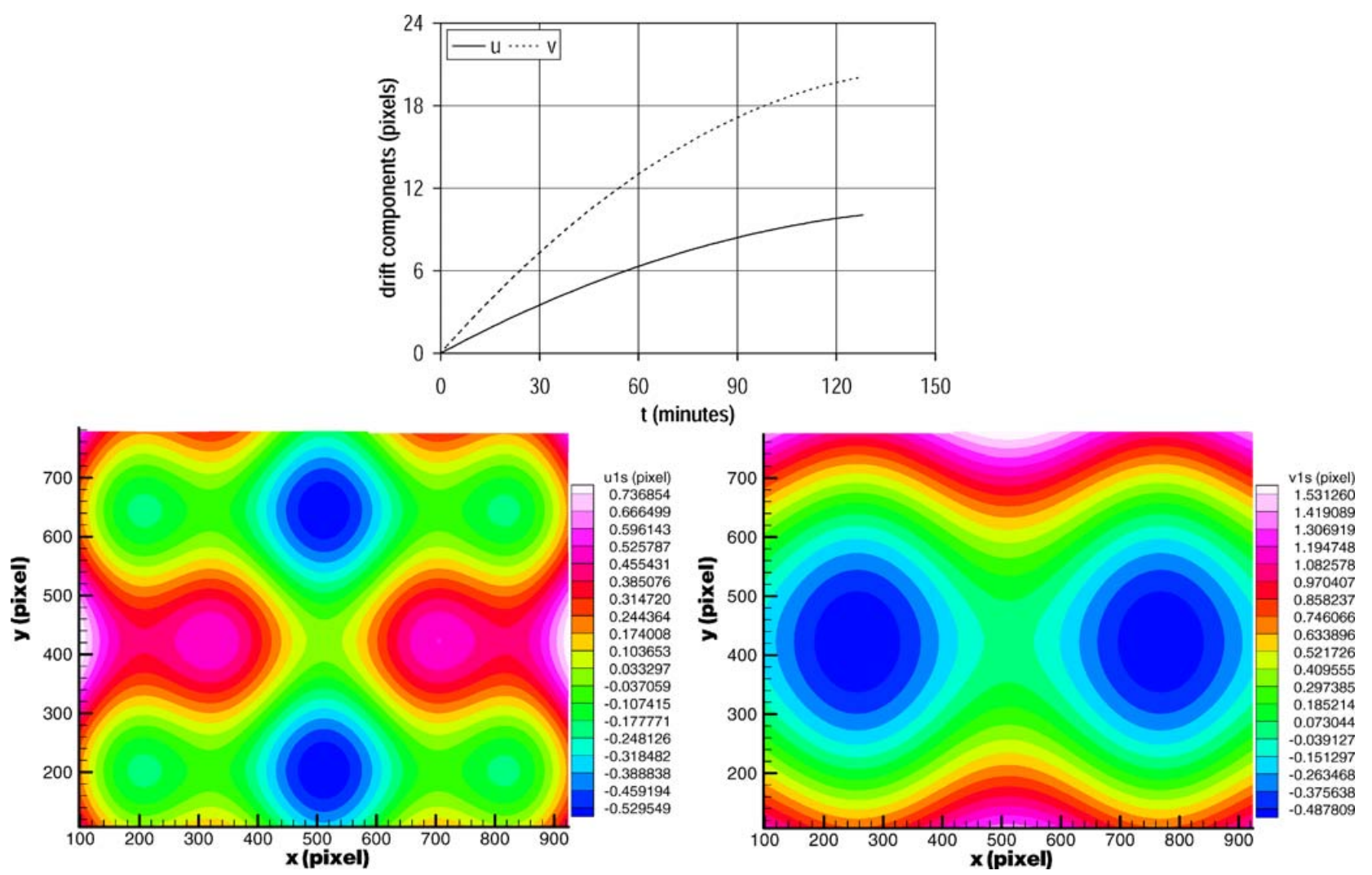

Fig. 4 Drift distortion and spatial distortion components for computer simulations. Spatial distortion ranges from 0.5 to +1.5 pixels within an image. Total drift displacements range up to 20 pixels over $120 \mathrm{~min}$

points at 30 specific times (corresponding to 15 image pairs in an experiment) are generated over a total time of $128 \mathrm{~min}$ Between each image pair, the effect of a cross shaped translation is included in the position of each point. To distort the position of each image point, the drift distortion function $\mathbf{D}_{\mathrm{dr}}(t)$ is assumed to have a quadratic form, the spatial distortion function is assumed to have the form of a combination of cosine wave and quadratic surface. Figure 4 shows both the drift distortion curves and the spatial distortion fields. With the inclusion of random error, the distorted positions of an image point $(X, Y)$ in image $n$ are written

$$
\begin{aligned}
\mathbf{R}(X, Y ; t)= & \mathbf{r}(X, Y ; t)+\mathbf{D}_{\mathrm{dr}}(X, Y ; t)+\mathbf{D}_{\mathrm{sp}}(X, Y) \\
& +\mathbf{G}(X, Y ; t)
\end{aligned}
$$

where $\mathbf{G}(X, Y ; t)$ is a Gaussian error function with mean value 0 and a prescribed standard deviation.

Instead of performing correlation, a total of $150 \times 120=$ 18,000 points with a spacing of 5 pixels and an initial position located at $(101,101)$ are calculated using equation (9). All 15 drift disparity maps are computed using this data.

\section{Simulation for the Calibration}

Figure 5 shows a direct comparison between the computed and the input drift at position $(561,646)$. Figure 6 shows the spatial distribution for the difference between the computed and the input vertical drift for the image acquired at $t=36 \mathrm{~min}^{7}$

After the correction to remove the effects of drift, the remaining disparity maps are obtained by subtracting the positions of points in the reference image, image 1 , from the positions of matching points in all other odd numbered images. The resulting disparity maps have the form

$$
\begin{aligned}
\operatorname{disp}_{\mathrm{sp}, n}(X, Y)= & \mathbf{D}_{\mathrm{sp}, n}\left(X+u_{n}, Y+v_{n}\right) \\
& -\mathbf{D}_{\mathrm{sp}, 1}(X, Y)+\mathbf{G}(X, Y ; t), \\
n= & 3,5, \ldots, N-1
\end{aligned}
$$

where $\left(u_{n}, v_{n}\right)^{T}$ is the rigid body motion of other images relative to image 1 .

By incorporating the disparity maps in equation (10), the procedure described in [5] is employed to perform least square bundle adjustment optimization and determine all translations and all parameters in the spatial distortion function. Figure 7 shows the difference between the computed and the input spatial distortion.

As a final check on the accuracy of the drift and spatial distortion correction method, the residual strain fields (with

\footnotetext{
${ }^{7}$ Since the form shown in equation (10) is independent of magnifi cation, all simulations are performed in pixels.
} 

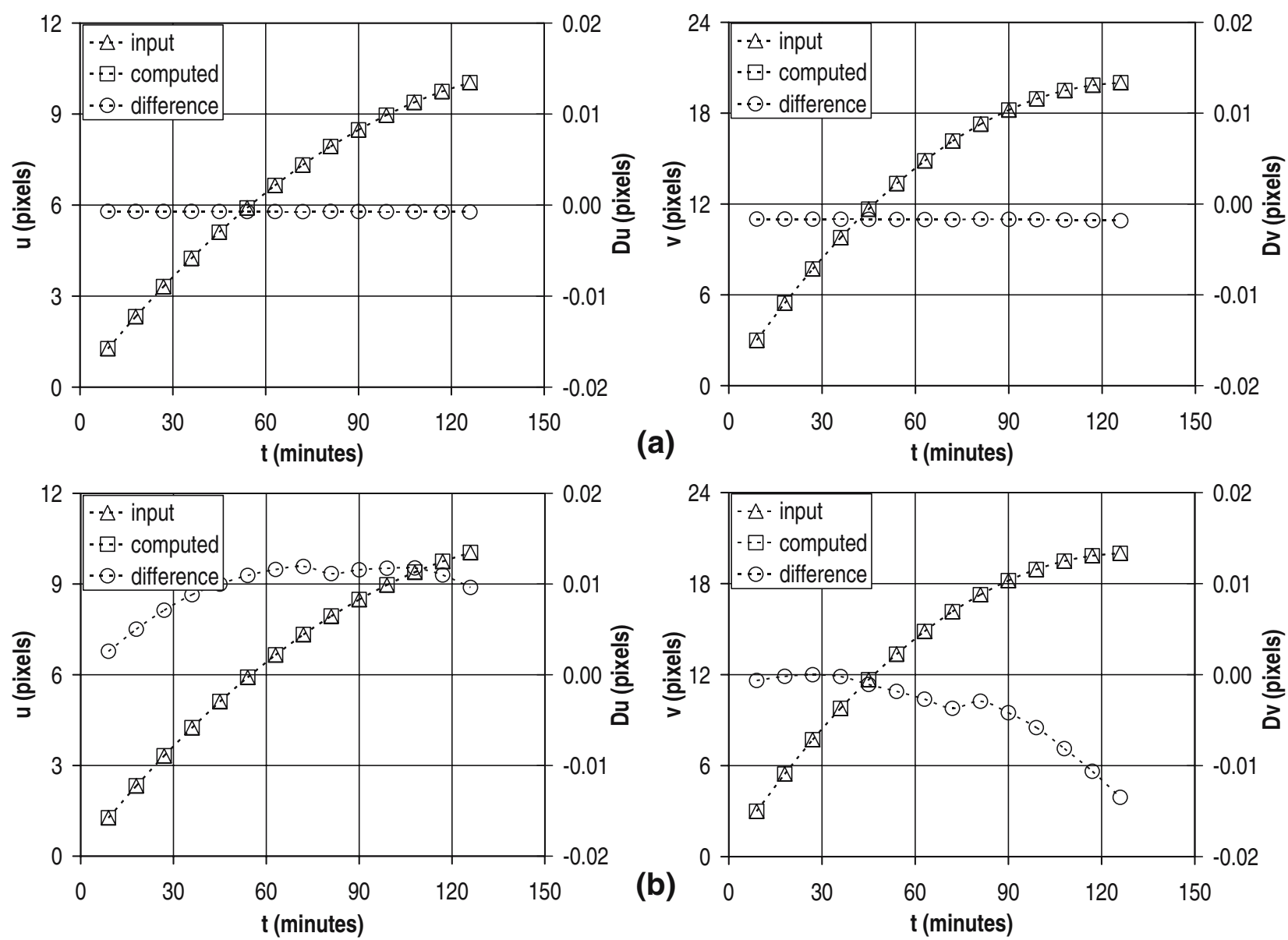

Fig. 5 Comparison of the computed and the input drift at pixel $(561,646)$ throughout calibration process (a) without Gaussian noise and (b) with Gaussian noise having standard deviation of 0.025 pixels added to the measurements

and without Gaussian noise in the displacement values) are computed for all of the images within the calibration phase. Without Gaussian noise in the displacement components, the computed strains are less than $1 \times 10^{6}$ throughout the entire sequence. With Gaussian noise having a standard deviation of 0.025 pixels in each displacement component,

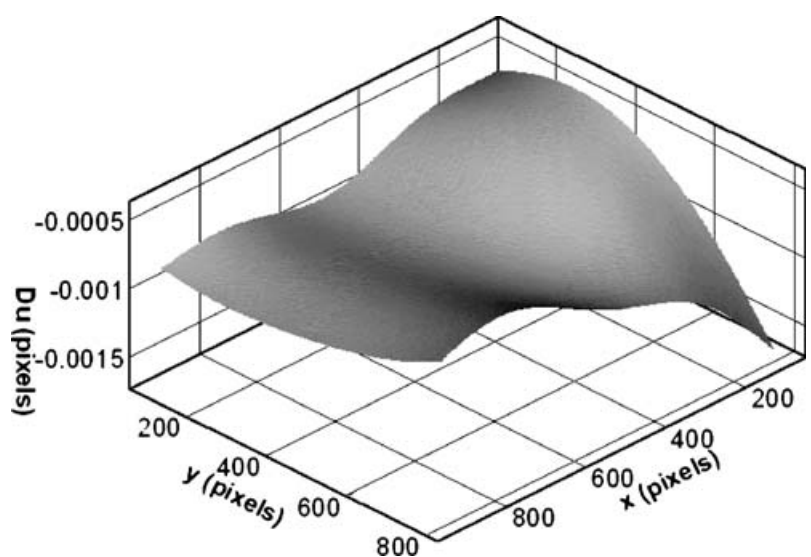

Fig. 6 Spatial distribution of difference between computed and input horizontal drift for the image acquired at $t 36 \mathrm{~min}$ all strains have an average strain between $\pm 6 \times 10^{5}$ and a standard deviation $\approx 5 \times 10^{5}$.

\section{Discussion}

The simulations assumed a total drift of 1020 pixels over $2 \mathrm{~h}$. Thus, between images in the sequence, the drift is relatively small and the local drift velocities can have considerable oscillation due to electro magnetic noise. Even so, the simulations confirmed that the method proposed will give good overall accuracy, even in the presence of substantial Gaussian noise in the measurements, when combining both drift and spatial distortion correction.

Since the drift is relatively small between images, it may appear that one can simply ignore this phenomenon. How ever, our simulations indicate that ignoring or incorrectly estimating drift distortion will introduce substantial errors in the spatial distortion correction. These errors will introduce large residual strains $(\approx 0.001)$ that cannot be removed from the data.

The primary emphasis in this work is to demonstrate the feasibility of extracting and removing drift and spatial 

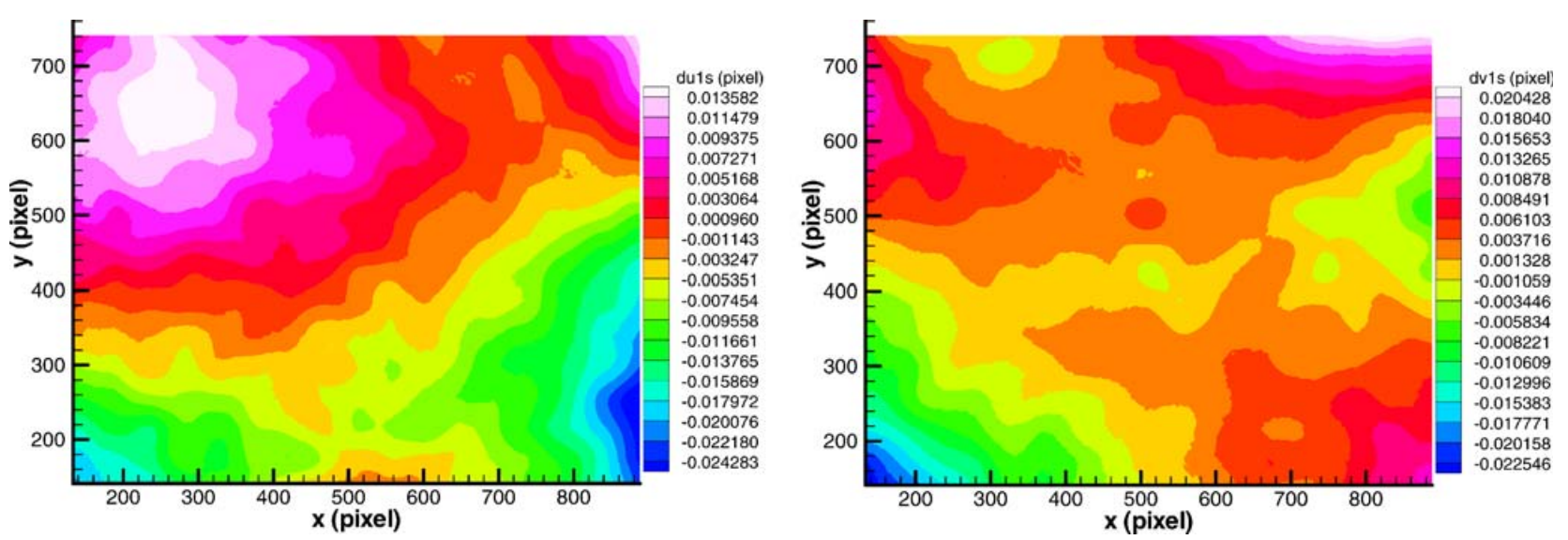

Fig. 7 Residual difference between the computed and the input spatial distortion. Gaussian noise with standard deviation of 0.025 pixels applied to input data. Residuals are less than \pm 0.02 pixels throughout the field

distortions. Though not discussed, separate studies also confirmed that the image magnification factors (i.e., the scale factors in pixel $/ \mathrm{mm}$ on the object) can also be extracted with relatively small errors. Here, two approaches are employed. First, with known translations, the pixel motion measured by image correlation using 15 fully corrected images is shown to be within $\pm 0.5 \%$ of the input value. Second, with a known distance between 9 points on the image, the pixel distances computed using a relatively crude image analysis provided by FEI using fully corrected images is shown to give magnification factors that are within $\pm 1 \%$ of the known values in all cases.

\section{Experiments}

\section{Experimental Setup}

All SEM imaging in this paper is performed in the Southeastern Electron Microscopy Center, University of South Carolina, using a FEI Quanta 200 SEM in the high vacuum mode with the BSE detector. The data acquisition process uses 8 bits to store each value of intensity in the image file. After all SEM working parameters are set and a clear image is captured on the screen, the initial images are not acquired until delaying by at least $15 \mathrm{~min}$ or more to avoid the observed large gradients that occur during initial transients.

For the e beam, (a) the accelerating voltage is $30 \mathrm{kV}$, (b) spot size is $3\left(\approx 4 \mathrm{~nm}\right.$ in diameter), and (c) dwell time $t_{D}=$ $10^{4} \mathrm{~s}$. The image array has a size of $1,024 \times 884$, this data corresponds to an image acquisition time $t_{F}=94.67 \mathrm{~s}$. It is noted that these SEM working parameters remain fixed for the $\times 200$ experiments. For the $\times 10,000$ experiments, image integration is performed with 16 scans combined to represent a single image; the total image acquisition time remains fixed at $t_{F}=75.68 \mathrm{~s}$ with all other parameters remaining the same.
The miniature rectangular tensile specimen is machined from AL2024 T4 sheet, with a length of $50 \mathrm{~mm}$, a width of $10 \mathrm{~mm}$ and a thickness of $1 \mathrm{~mm}$. The specimen is machined in the L T orientation; the rolling direction is along the loading direction. The grain structure in the rolled sheet is characterized as "elongated pancake grains," with grain thickness of $30 \mu \mathrm{m}$ and in plane dimensions ranging up to $1 \mathrm{~mm}$ in extent along the rolling direction.

Figure 8 shows a top view of the miniature commercial tensile loading frame used in this study; the frame has a total weight of $2.3 \mathrm{~kg}$ and a load capacity of $4,300 \mathrm{~N}$ in the configuration shown. The frame is installed on the SEM translation stage in the chamber. To stabilize the relatively heavy load frame, the $\mathrm{Z}$ translation portion of the SEM stage is removed.

For comparison to the image correlation based measure ments, a single strain gage of length $6.35 \mathrm{~mm}$ is aligned with the loading direction and bonded to the back side of the specimen near mid length using standard experimental

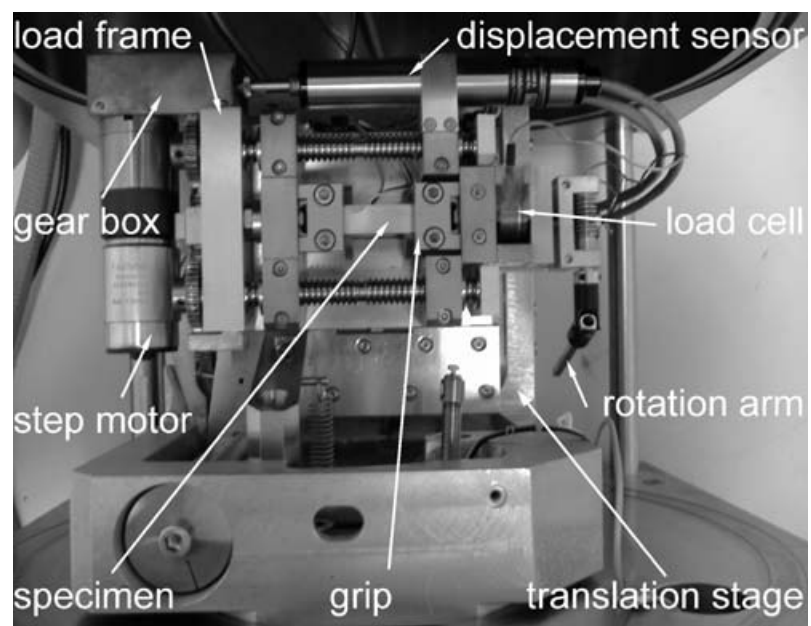

Fig. 8 Top view of the miniature tensile loading frame 
Fig. 9 SEM BSE images of (a) lithographically applied gold pattern on aluminum surface for $\times 200$ magnification and (b) rearranged gold pattern on aluminum surface for $\times 10,000$ magnification
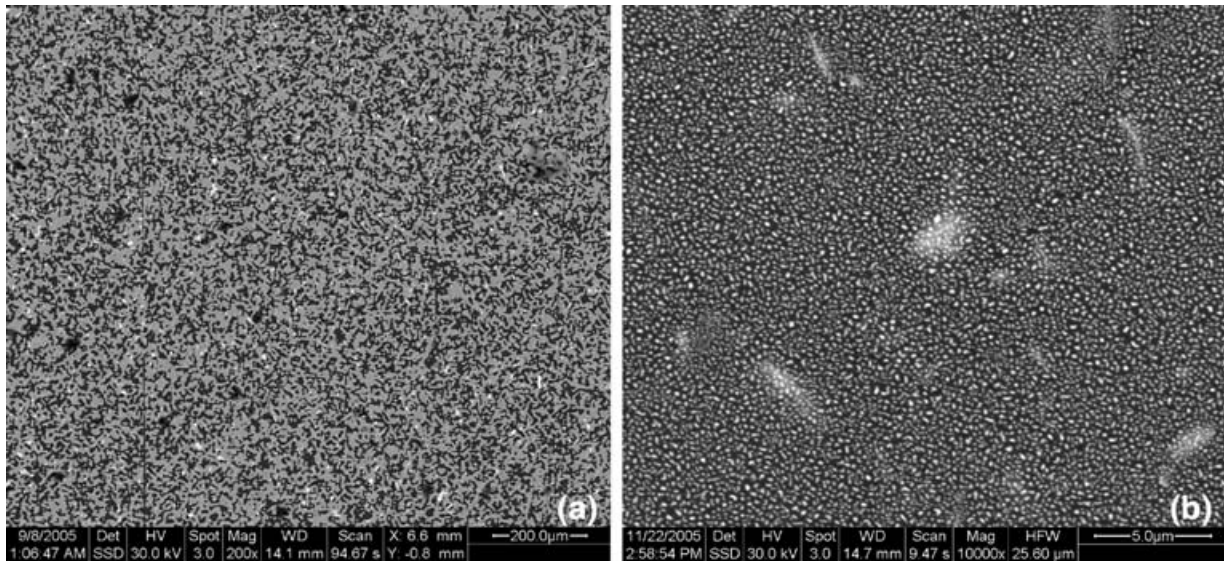

techniques. The lead wires are routed outside the SEM chamber via a wire port for attachment to a readout unit.

\section{$\times 200$ Tensile Loading Experiment}

At the SEM magnification of $\times 200$, each pixel corresponds to $\approx 1.25 \mu \mathrm{m}$ on the object. The working distance is $14.1 \mathrm{~mm}$ due to the configuration of the loading frame. Figure 9(a) shows an SEM image of the gold patterned aluminum specimen at $\times 200$. The pattern is applied using lithographic methods described in previous work [23 25]; the average gold speckle size is $8 \mu \mathrm{m}$.

Calibration, translation and strain experiments are performed during one experimental session. To perform the calibration process described in "Imaging Model for Planar Object," Section all orthogonal translations during the calibration are performed manually via external motion controls. The translation experiment adds additional trans lated images (no strain) after completing the calibration sequence. The strain experiment adds a series of images of
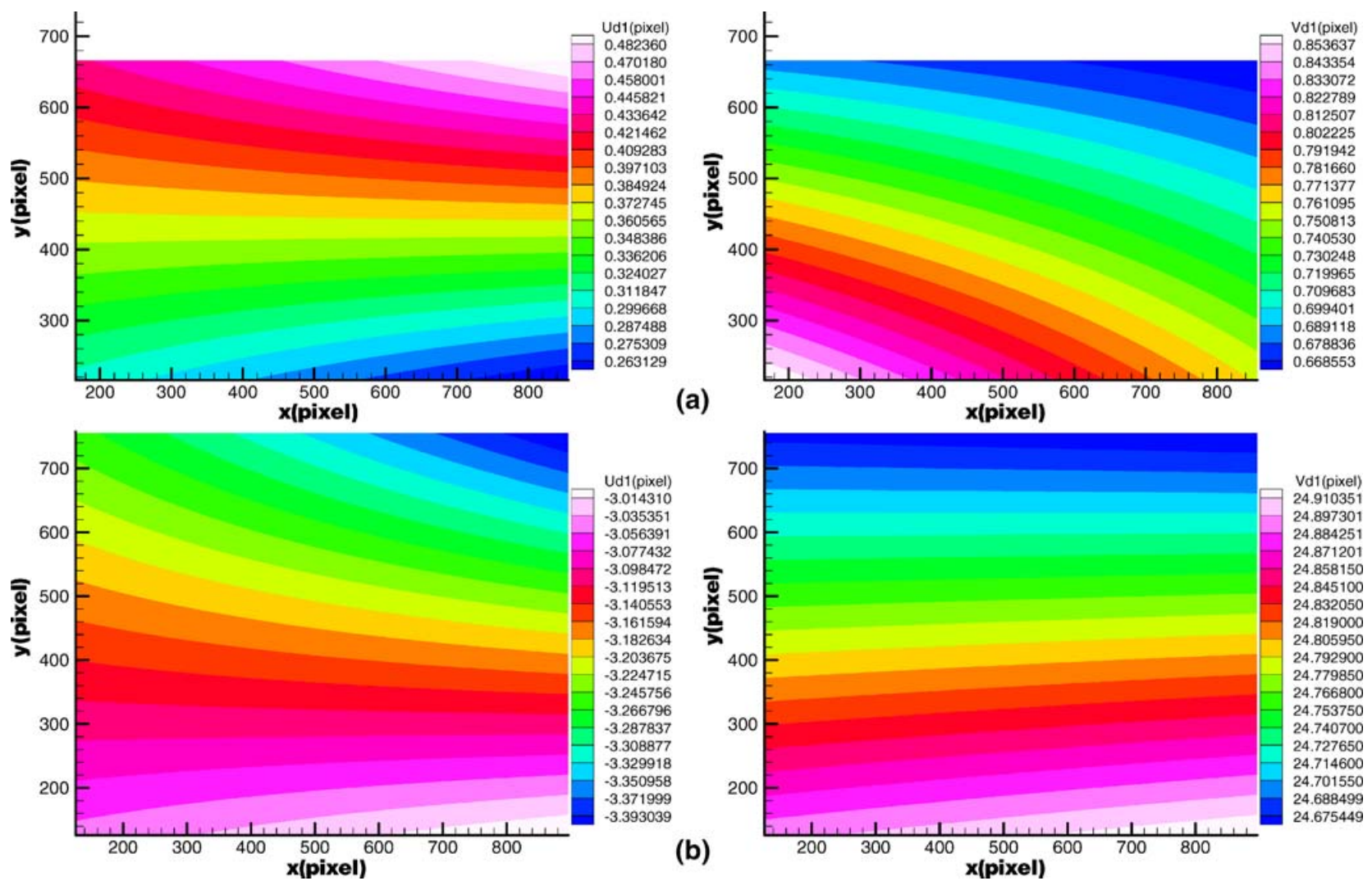

Fig. 10 Typical drift distortion functions for (a) $\times 200$ magnification at time $t 36 \mathrm{~min}$ and (b) $\times 10,000$ magnification at time $t 30 \mathrm{~min}$ 

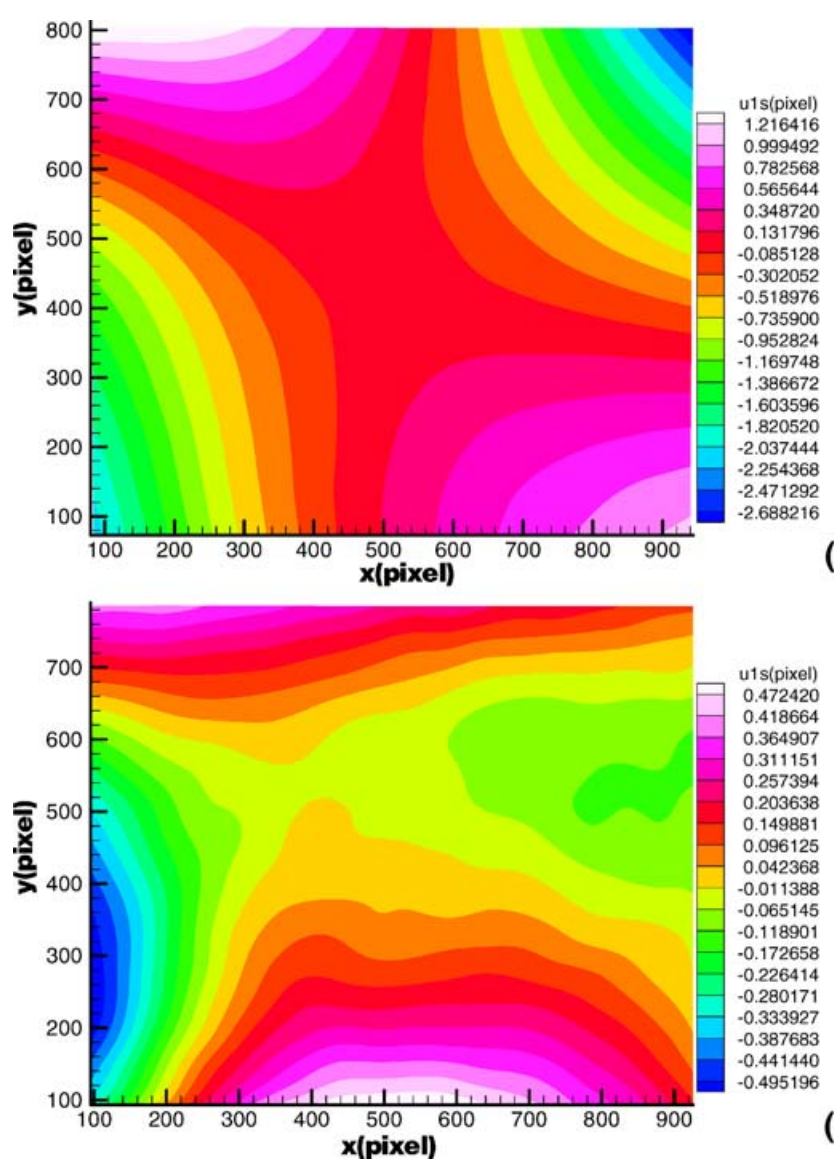

Fig. 11 Measured horizontal and vertical spatial distortion fields in an FEI Quanta 200 SEM at magnifications of (a) $\times 200$ and (b) $\times 10,000$

the specimen undergoing uniaxial loading that is performed in load control using a Lab view program.

After completing all phases of the experiment and acquiring all of the images, the displacement fields (disparity maps) are obtained by 2D DIC using the commercial software VIC $2 \mathrm{D}{ }^{8}$ The procedures outlined previously are employed to determine the drift and spatial distortion functions. Unless otherwise noted, all image correlations are performed at $150 \times 120$ pixel locations using a $43 \times 43$ pixel subset size and spacing between subset centers of 5 pixels; the first subset center is at $(101,101)$. Thus, all disparity maps contain 18,000 points.

\section{Calibration and distortion removal}

A cross shaped motion path is performed with eight horizontal and eight vertical motions. Figure 10(a) shows the measured drift displacement field at $t=36 \mathrm{~min}$ Figure 11(a) shows the measured spatial distortion correc

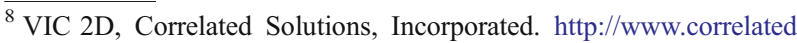
solutions.com.
}
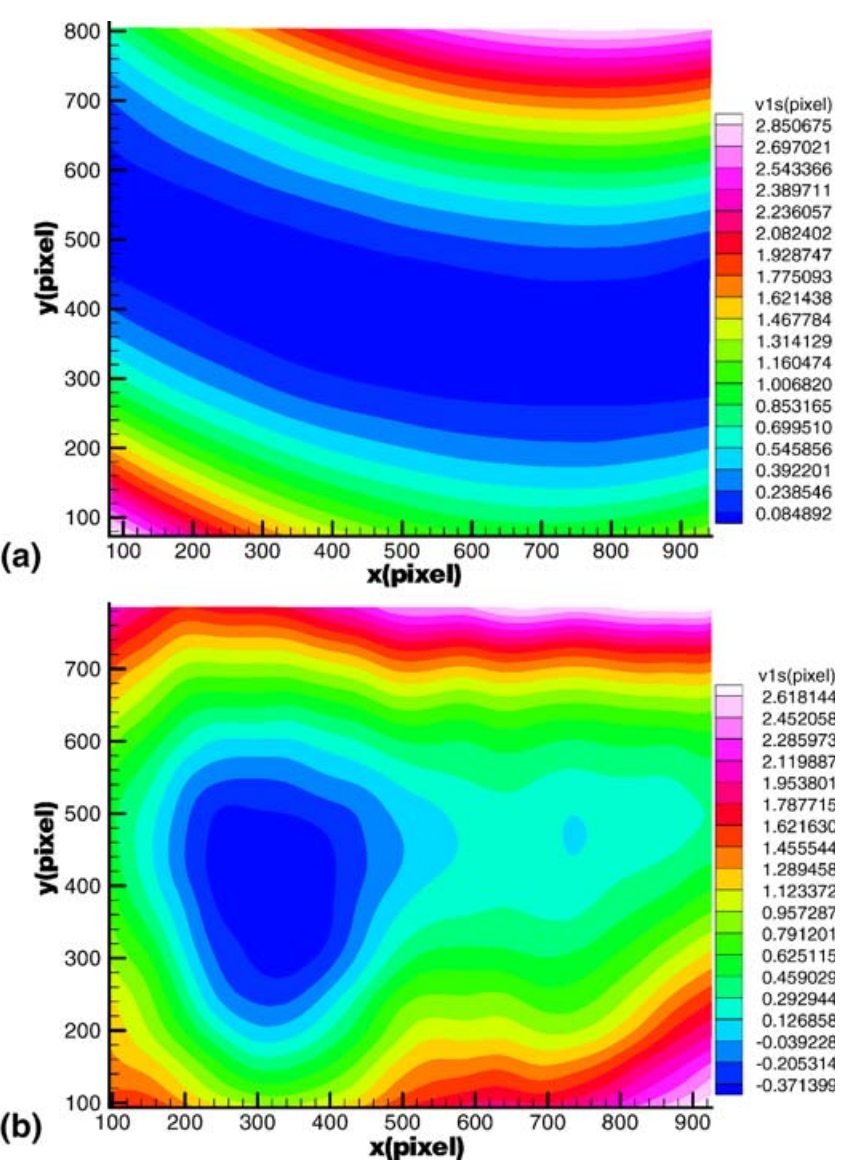

tion functions for both horizontal $(u)$ and vertical $(v)$ displacement fields after completing the calibration process. Here, it is clear that the spatial distortions are much larger than the measured drift distortions at this magnification, with spatial distortion corrections up to 2 pixels in the $y$ direction and up to 1 pixel in the $x$ direction.

After correcting all of the calibration images for both drift and spatial distortions, the displacement gradients are computed and the strain fields determined in each of the 16 calibration images. Figure 12(a) shows that the fully corrected $\varepsilon_{x x}, \varepsilon_{x y}$ and $\varepsilon_{y y}$ fields had average values near zero and a standard deviation of $6 \times 10^{5}$.

\section{Translations}

After completing the calibration sequence, the specimen is subjected to two additional in plane translations. To obtain the drift components during the post calibration portion of the experiment, the two disparity maps are used with the disparity maps in the calibration portion to extract the drift corrections. Since the spatial distortion function is the same as computed previously, the new local drift function and the spatial distortion function are used to correct all positions in both pairs of images. 

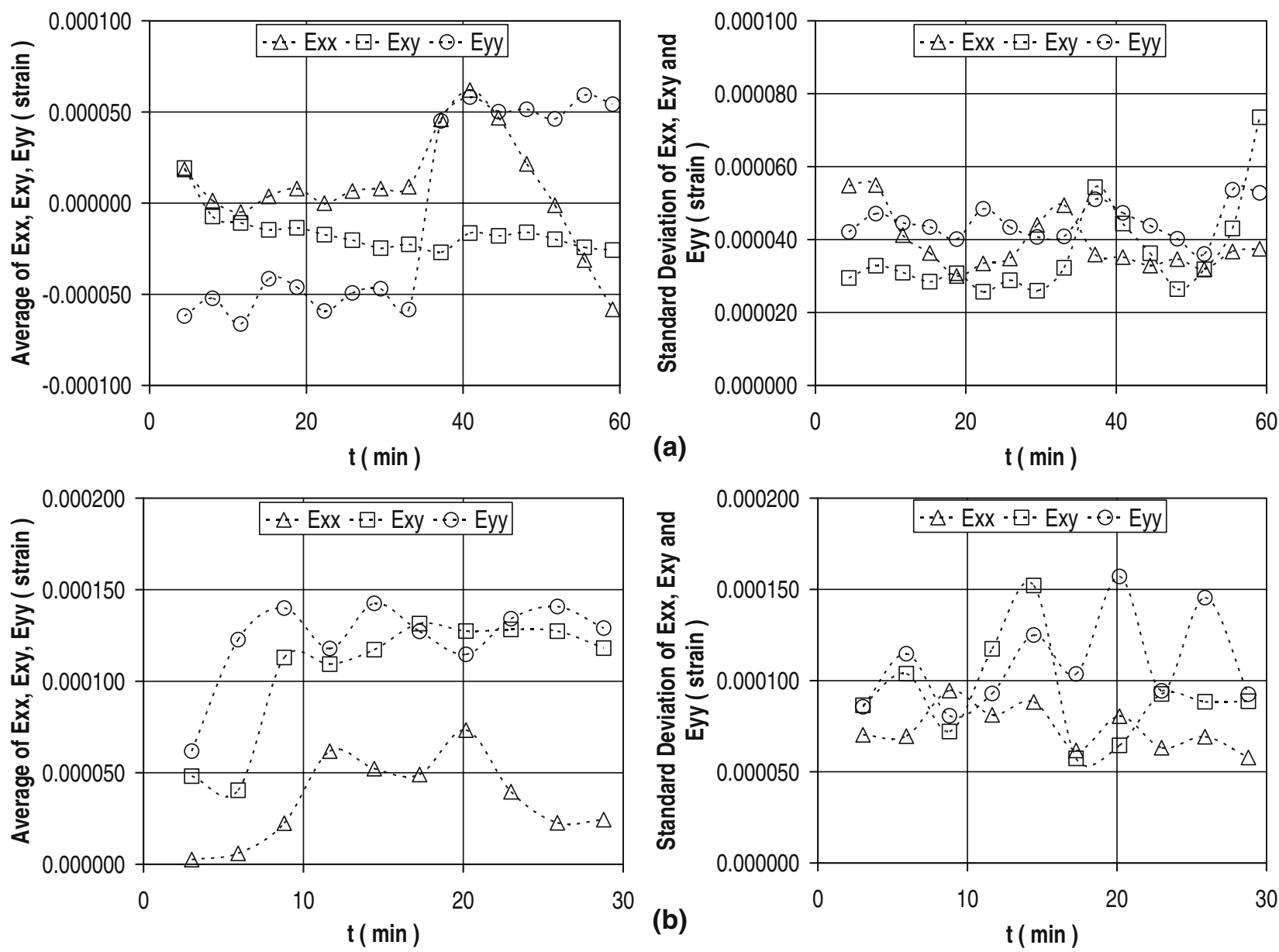

Fig. 12 Averaged strain with standard deviation for the calibration images after both drift and spatial distortion correction at (a) $\times 200$ magnification and $(\mathbf{b}) \times 10,000$ magnification

After correcting the disparity data for drift and spatial distortions, experimental data indicates that the strain fields have a mean value near zero with a standard deviation of $7 \times 10^{5}$, confirming that the distortion correction approach can be used effectively without altering the accuracy of the measurements.

\section{Uniaxial strain}

After completing the translation sequence, the specimen is subjected to six additional strain levels in uniaxial tension. In this study, a total of 14 images (seven pairs) are acquired, with the first two used as reference images. ${ }^{9}$ To obtain the additional drift distortions that accumulated during the strain portion of the experiment, the disparity maps obtained by comparing the images within each pair are

\footnotetext{
${ }^{9}$ To minimize the potential for unwanted additional image motion after loading the specimen, a hold time of $30 \mathrm{~s}$ is maintained prior to acquiring each additional image pairs.
}

measured and used to determine the extended drift correction for the strain images. The spatial distortion function obtained during calibration and the new local drift function obtained for the set of strain images are used to correct all spatial positions.

Figure 13(a) presents the average strain data and the standard deviation for $\varepsilon_{x x}, \varepsilon_{x y}$ and $\varepsilon_{y y}$ at all loading levels. Figure 14 compares the average axial strain data with strain gage results. The Young's modulus obtained from the strain gage measurements and the fully corrected image correla tion data is nearly the same, $71.1 \mathrm{GPa}$. Also shown in Fig. 14 is a plot of $\left|\varepsilon_{x x}\right| / \varepsilon_{y y} v s \varepsilon_{y y}$. The estimated value for Poisson's ratio is $\approx 0.33$, which is consistent with the range 0.280 .35 noted in the literature.

\section{$\times 10,000$ Tensile Loading Experiment}

The magnification of $\times 10,000$ in our FEI Quanta 200 SEM corresponds to $\approx 0.5 \mathrm{~nm}$ per pixel and an imaging window of about $25.6 \times 22.1 \mu \mathrm{m}$ with a working distance of $14.7 \mathrm{~mm}$. Following the procedures described in Part I, 

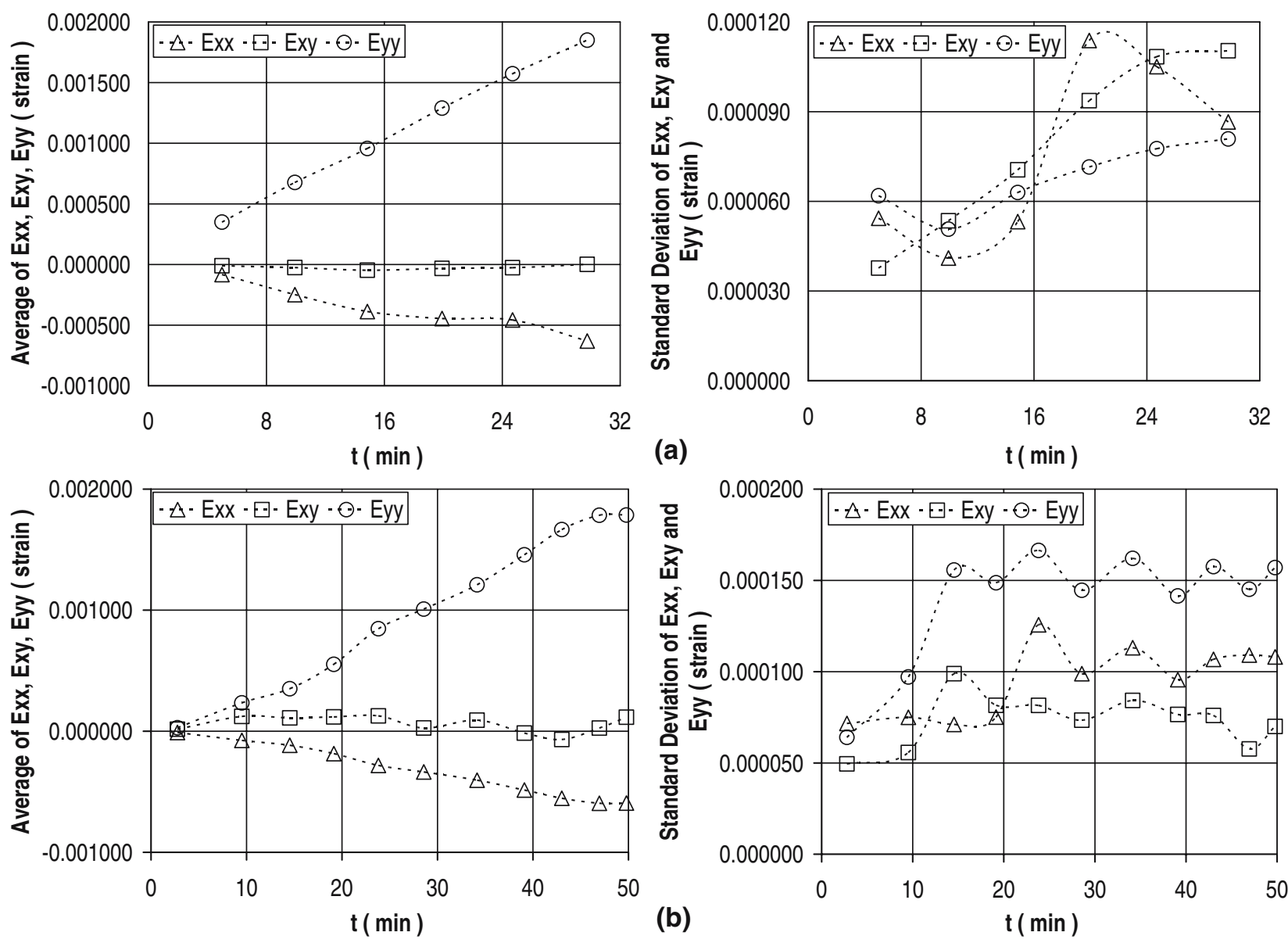

Fig. 13 Averaged strain with standard deviation on images for measurement phase after both drift and spatial distortion correction at (a) $\times 200$ magnification and $(\mathbf{b}) \times 10,000$ magnification

image integration was used to record each image, with 16 scans performed to acquire each image.

To have much smaller size of gold pattern on the aluminum specimen, the evaporation coating technique [26] is used instead of the photo resistance development technique for the $\times 200$ pattern. Briefly, a thin gold coating is re arranged to develop the appropriate random pattern for
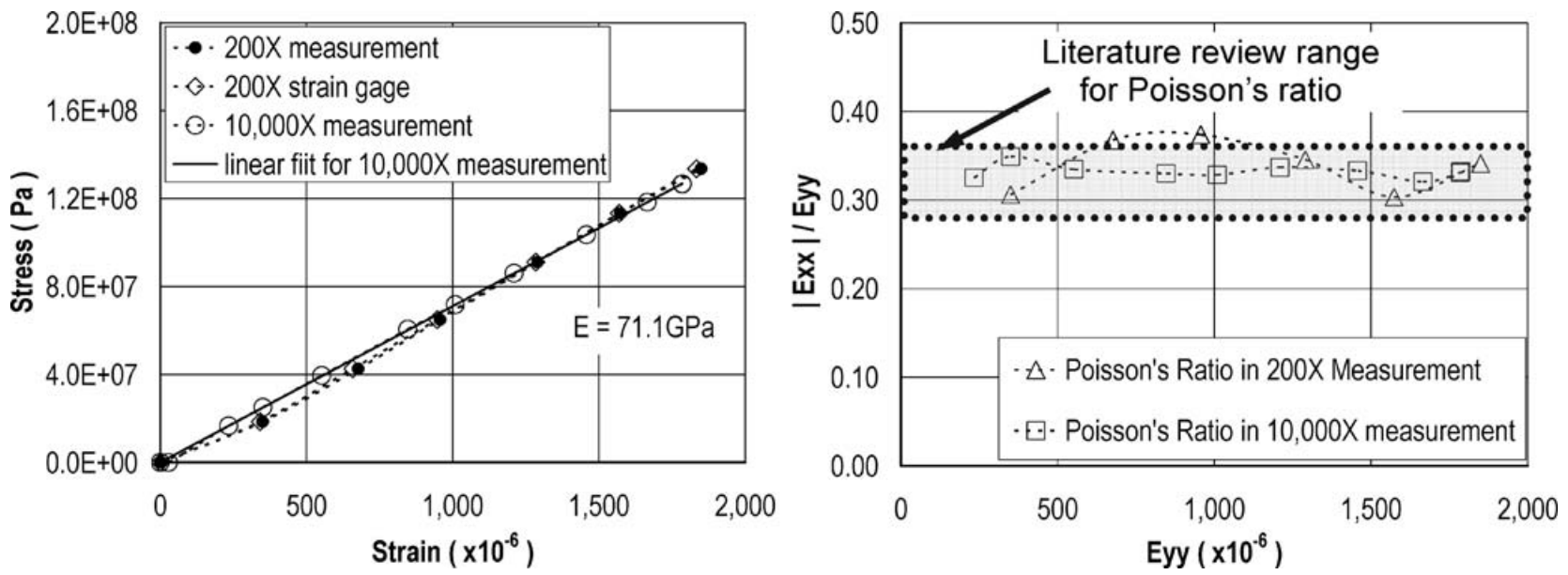

Fig. 14 Comparison of DIC based estimates for Young's Modulus and Poisson's Ratio to literature values for data obtained at magnifications of $\times 200$ and $\times 10,000$ 


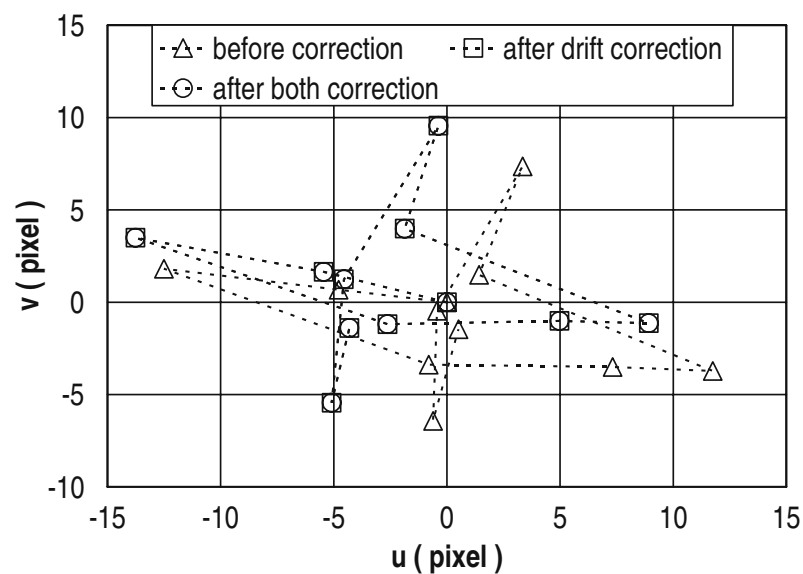

Fig. 15 Actual and corrected translation path during calibration at $\times 10,000$ magnification

the image correlation. Figure 9(b) shows the re arranged gold pattern on the specimen surface.

\section{Calibration and distortion removal}

In the calibration phase, a series of 11 image pairs are acquired using horizontal and vertical translations. Since translations should be relatively small (less than 10 pixels, total of all translations less than \pm 50 pixels in each direction), to minimize the amount of image that moves outside field of view, translations are performed using an internal SEM function.

Figure 15 shows the cross shaped motion path before and after performing drift and spatial distortion corrections. The fact that translations composing the actual path are not truly orthogonal reflects inaccuracies associated with application of the SEM positional function. These inaccu racies resulted in unwanted directional shifts during the translation process and will affect the accuracy of the spatial distortion estimation which assumes either horizon tal and vertical motions in the sequence.

Figure 10(b) shows the measured drift displacement field at $t=30 \mathrm{~min}$ It is noted that the measured drift distortion fields are relatively large, especially for the vertical displacement that is perpendicular to the scan line to form the image. The 0.230 .38 pixels non uniform drift in this case will still add at least $2.6 \times 10^{4} 3.7 \times 10^{4}$ error to any measurement unless corrected. Furthermore, if the drift is too large, then the overlapped area may decrease so much that 2D DIC will not have sufficient area to obtain deformation fields. ${ }^{10}$

Figure 11(b) shows the measured spatial distortion correc tion functions for both horizontal $(u)$ and vertical $(v)$

\footnotetext{
${ }^{10}$ As is discussed in detail in "Discussion" and shown in Fig. 16, the average drift displacement will couple with the spatial distortion field and introduce large spatial distortions.
}

displacement fields after completing the calibration process, with corrections in the range of 0.50 .5 pixels for horizontal displacement and 0.42 .6 pixels for vertical displacement.

As shown in Fig. 12(b), the fully corrected averaged strain components for the calibration images, $\varepsilon_{x x}, \varepsilon_{x y}$ and $\varepsilon_{y y}$, are below $1.5 \times 10^{4}$ and the standard deviation for each component is less than $1.5 \times 10^{4}$. Though somewhat higher than measured at $\times 200$ primarily due to inaccuracies in the spatial distortion process, the values are quite good and confirm that elastic deformations can be measured using an SEM for imaging.

\section{Uniaxial strain}

There are nine steps in the tensile loading experiment, with one additional pair of images before loading and after loading to the maximum value. Similar to the $\times 200$ experiments, images are acquired $30 \mathrm{~s}$ after the load is increased to the required value.

Figure 13(b) presents the average strain data and standard deviation data for $\varepsilon_{x x}, \varepsilon_{x y}$ and $\varepsilon_{y y}$ for all load ing levels, Fig. 14 presents a direct comparison between (a) uniaxial stress strain data and (b) Poisson's ratio results for both $\times 200$ and $\times 10,000$.

\section{Discussion}

As shown in Figs. 10 and 11, the drift and spatial distortion correction functions at $\times 200$ and $\times 10,000$ are different. Even with these differences, the distortion correction procedures implemented in this study are sufficiently robust to extract them and remove the errors. Thus, the data clearly indicates that basic elastic material properties can be reasonably quantified using digital image correlation with corrected SEM images. Even at low magnification, both corrections are essential for accurate measurement of elastic response.

To quantify the importance of correcting for spatial distortion when measuring small deformations, a series of numerical studies were performed using the spatial distor tion fields for $\times 10,000$ shown in Fig. 11(b); similar results were obtained at $\times 200$. Assuming horizontal and vertical translations of 5 pixels between uncorrected images, the gradient in the spatial distortion field is used to estimate the distortion induced strain errors that would occur throughout the field of view. The strain errors due to these small translations of the images are shown in Fig. 16. As shown in Fig. 16, the distribution in strain errors, $E \varepsilon$, is relatively complex, ranging from $-1.5 \times 10^{4} \leq E \varepsilon_{x x} \leq+1 \times 10^{4}$, $-3.3 \times 10^{4} \leq E \varepsilon_{x y} \leq+4.3 \times 10^{4}$ and $-4 \times 10^{4} \leq$ $E \varepsilon_{y y} \leq+6 \times 10^{4}$. It is important to note that these errors increase with image translation, with the maximum $E \varepsilon_{y y}$ 


\section{$\mathrm{U}=\mathbf{5}$ pixels}
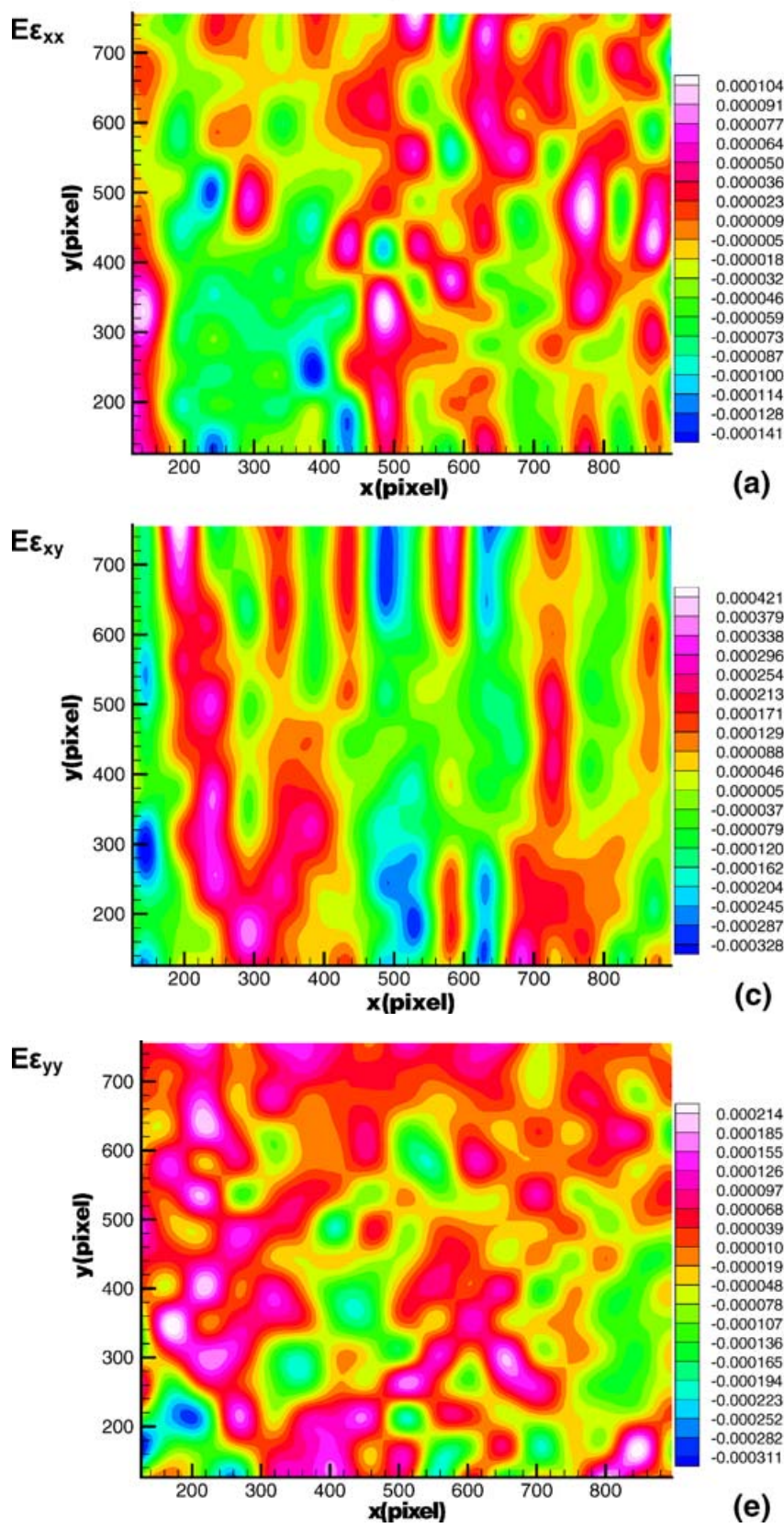

(a)
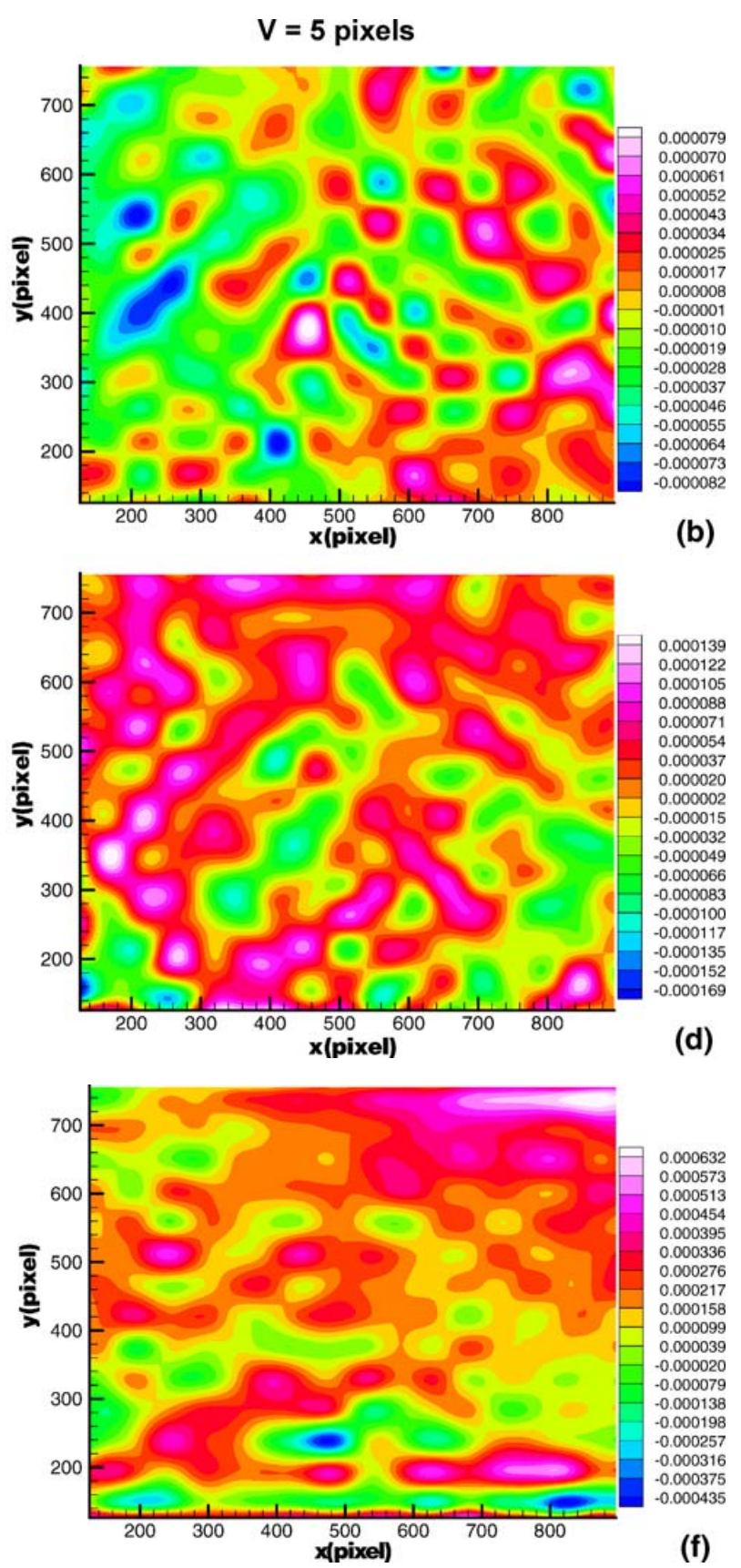

Fig. 16 Strain error fields, $E \varepsilon$, measured when uncorrected images are translated five pixels horizontally $(U)$ and vertically $(V)$. Errors estimated using gradients of measured spatial distortion field

exceeding $+1 \times 10^{3}$ for an 8 pixel translation. Furthermore, since temporally varying drift will introduce image trans lations, coupling exists at all times between spatial and drift distortions and confirms the importance of drift distortion removal for accurate, image based deformation measurements. For magnifications ranging from $\times 100$ to $\times 500$, the noise in SEM images has been shown to introduce random displacement variations in the image correlation measure ments $(43 \times 43$ subsets, subset center spacing of 5 pixels $)$ with a range \pm 0.1 pixels. Using Gaussian noise with this range, our studies have shown that the noise can be removed from the measurements using a Butterworth Filter with a spatial cutoff wavelength equal to half of the subset size (22 pixels), without decreasing the spatial frequency content of the underlying displacement measurements. Specifically, after using the Butterworth Filter the standard deviation in the measured strains is less than $1 \times 10^{4}$ for all components with a spatial resolution of 43 pixels for the 
measurements, the same spatial resolution expected using a $43 \times 43$ subset. Thus, the results confirm that distortion correction algorithms and data reduction are effective in converting the SEM into a quantitative measurement system for either elastic or elastic plastic deformations.

Inspection of the disparity maps obtained at $\times 10,000$ shows clearly an increase in variability relative to data obtained at $\times 200$. Specific reasons include (a) inaccuracies in the calibration path directions, (b) non optimal density of random pattern, (c) image contrast, and (d) image stability over time. Improvements in each of these areas should increase the accuracy of measurements at high magnification.

Initial experimental results at $\times 10,000$ indicate (a) displace ment noise levels in the range \pm 0.2 pixels which is approxi mately double the range obtained at $\times 200$, and (b) high frequency variations in displacement that are similar to that obtained at $\times 200$. These results suggest that the strains will have a standard deviation $\approx 1.2 \times 10^{4}$ with a spatial resolution of 43 pixels, implying that the Butterworth Filter for noise removal can be extended to high magnification for metrolog ical measurements.

The measured drift function at $\times 10,000$ is 1 to 2 orders of magnitude larger than seen at $\times 200$, with the component in the direction perpendicular to each scan line having a different trend. Even with these differences, the general methodology has been shown to be effective for removing drift distortions at all magnifications.

Given the wide range of SEM systems available today, it is worth noting several points regarding this issue. First, the Quanta 200 SEM used in these studies employs a tungsten filament for electron emission, maintaining a voltage on the anode throughout the imaging process, an option that is known to improve the accuracy and repeatability of the e beam scan.

Second, though Field Emission Guns (FEGs) are generally perceived to be more stable than Tungsten Electron Guns (TEGs), it is known that cold FEGs are intrinsically less stable because they are "flashed" prior to use each day; this rapid heat cool cycle tends to introduce creep in the tip, a process that may last an hour or more, resulting in a significant impact on imaging stability.

Third, the stability of a system may be a function of the settings employed during the imaging process. For exam ple, we observed defocus and increased drift during one set of experiments using an FEG, with at least a portion of the instability attributed to a low temperature setting on a cold water source. $^{11}$

\footnotetext{
${ }^{11}$ Considerable insight into the key aspects of modern SEM systems is provided by Prof. David Joy, UT/ORNL Distinguished Scientist, University of Tennessee at Knoxville, and an international expert in SEM physics and system construction.
}

\section{Concluding Remarks}

In sharp contrast with the approach of early SEM measure ments, where the investigators simply accepted the accura cy obtainable and successfully performed their studies for important problems amenable to such limitations, this work presents and validates a general approach that successfully extends the range of measurements obtainable in an SEM to the small deformation (elastic) regime so that full elastic plastic deformation studies can be performed in an SEM.

The novel method outlined in this work relies on a combination of drift distortion correction and a priori spatial distortion correction so that accurate elastic and elastic plastic deformation measurements can be obtained using SEM images; both corrections are essential to obtain accurate deformation measurements throughout the field.

Our experimental studies have clearly shown the importance of the quality of both the microscope translation stage used for calibration movements and the miniature tensile loading system. In most SEM systems, (a) transla tion stage movements are performed by click and drag processes that are relatively inaccurate, (b) translation stage control systems are generally prone to backlash and/or overshoot, and (c) stages generally have few features available to maintain constant height and orientation of the stage; \pm 0.5 degrees is a typical accuracy for rotational position on most SEM systems. Given these issues, it is essential that baseline studies be performed to identify potential problems prior to performing the critical experi ments. Regarding the miniature loading frame used to load the specimen, issues such as specimen misalignment, inadequate specimen gripping, imprecise loading and/or applied displacement and instability of loading platform within the SEM must be addressed and resolved to ensure repeatability in the $2 \mathrm{D}$ measurements.

Simulation results have shown that typical drift process es in an SEM can be adequately reconstructed using local drift velocity measurements. However, if higher gradients in drift are present during the early stages of image acquisition, the simulations also show that image acquisi tion time should be reduced and additional images acquired during this period for accurate drift reconstruction, a situation that may not be feasible with a given microscope. In practice, image acquisition should be conducted 15 $30 \mathrm{~min}$ after the first SEM scans are initiated so that the gradients in drift are reduced to a more manageable level.

Acknowledgements The support of (a) the National Science Foundation, Dr. Oscar Dillon and Dr. Kenneth Chong through grant CMS 0201345, (b) Intel and Mr. Michael Mello through fund DOCC 22860, (c) the Army Research Laboratory and Dr. Adam Rawlett through Cooperative Agreement W911NF0420026, and (d) the Ecole des Mines d'Albi (France) who provided a grant for Nicolas Cornille 
is gratefully acknowledged. The technical assistance provided by Mr. Dana Dunkelberger and Dr. Donggao Zhao in the Southeastern Electron Microscopy Center, as well as their financial support of this research, is deeply appreciated.

\section{References}

1. Beyer HA (1992) Accurate Calibration of CCD cameras. Interna tional Conference on Computer Vision and Pattern Recognition (CVPR'92). Champaign Urbana, IL, USA, pp 96101 June.

2. Weng J, Cohen P, Herniou M (1992) Camera calibration with distortion models and accuracy evaluation. IEEE Trans Pattern Anal Mach Intell 14(10):965 980

3. Hemmleb M, Albertz J, Schubert M, Gleichmann A, Köhler JM (1996) Digital microphotogrammetry with the scanning electron microscope. XVIII ISPRS Congress, Commission V, Vienna, Austria, July.

4. Doumalin P (2000) Microextensométrie locale par corrélation d'images numériques. $\mathrm{PhD}$ thesis, Ecole Polytechnique (France).

5. Schreier HW, Garcia D, Sutton MA (2004) Advances in light microscope stereo vision. Exp Mech 44(3):278 288.

6. MeX software. Alicona imaging. http://www.alicona.com.

7. SAMx. 3D TOPx package. http://www.samx.com.

8. Lacey AJ, Thacker NA, Yates RB (1996) Surface approximation from industrial SEM images. British Machine Vision Conference (BMVC'96), 725734.

9. Agrawal M, Harwood D, Duraiswami R, Davis L, Luther P (2000) Three dimensional ultrastructure from transmission electron $\mathrm{mi}$ croscope tilt series, 2nd Indian Conference on Vision, Graphics and Image Processing (ICVGIP 2000). Bangalore, India.

10. Vignon F, Le Besnerais G, Boivin D, Pouchou JL, Quan L (2001) $3 \mathrm{D}$ reconstruction from scanning electron microscopy using stereovision and self calibration. Physics in signal and image processing, Marseille, France, June.

11. Sinram O, Ritter M, Kleindiek S, Schertel A, Hohenberg $H$, Albertz J (2002) Calibration of an SEM, using a nano positioning tilting table and a microscopic calibration pyramid. ISPRS Commission V Symposium, Corfu, Greece, pp 210215.

12. Doumalin P (2000) Microextensométrie locale par corrélation d'images numériques. $\mathrm{PhD}$ thesis, Ecole Polytechnique.

13. Hemmleb M, Albertz J (2000) Microphotography the photo grammetric determination of friction surfaces. IAPRS XXXIII, Amsterdam, The Netherlands.

14. Lee HS, Shin GH, Park HD (2003) Digital surface modeling for assessment of weathering rate of weathered rock in stone monu ments. The International Archives of the Photogrammetry, Remote Sensing and Spatial Information Sciences, Ancona, Italy, July.
15. Bi H, Hartsough C, Han B (2006) Nano pattern recognition and correlation technique for deformation measurement of nano structures. 7th International Symposium on MEMS and Nano technology (7th ISMAN), St. Louis, MO, USA, pp 148149 June.

16. Sutton MA, Li N, Garcia D, Cornille N, Orteu JJ, McNeill SR, Schreier HW, Li XD (2006) Metrology in an SEM: theoretical developments and experimental validation. Meas Sci Technol 17:2613 2622 .

17. Ravn O, Andersen NA, Sorensen AT (1993) Auto calibration in automation systems using vision. 3rd International Symposium on Experimental Robotics (ISER'93), Kyoto, Japan, pp 206218 October.

18. Lavest JM, Viala M, Dhome M (1998) Do we really need an accurate calibration pattern to achieve a reliable camera calibra tion? 5th European Conference on Computer Vision (ECCV'98), Freiburg, Germany, pp 158 174, June.

19. Brown DC (1971) Lens distortion for close range photogramme try. Photometric Engineering 37(8):855 866.

20. Peuchot B (1993) Camera virtual equivalent model 0.01 pixel detector. Comput Med Imaging Graph 17(4):289 294.

21. Brand R, Mohr P, Bobet P (1994) Distorsions optiques: corrections dans un modéle projectif; 9th Congress AFCET, Reconnaissances des Formes et Intelligence Articielle (RFIA'94), Paris, France, pp 87 98, January.

22. Cornille N (2005) Accurate 3D shape and displacement measure ment using a scanning electron microscope. $\mathrm{PhD}$ thesis, Ecole des Mines d'Albi (France) / University of South Carolina.

23. Collette SA, Sutton MA, Miney P, Reynolds AP, Li XD, Colavita PE, Scrivens WA, Luo Y, Sudarshan T, Muzykov P, Myrick ML (2004) Development of patterns for nanoscale strain measure ments: I. Fabrication of imprinted $\mathrm{Au}$ webs for polymeric materials. Nanotechnology 15:1812 1817.

24. Sutton MA, Zhao W, McNeill SR, Helm JD, Piascik RS, Riddell WT (1999) Local crack closure measurements: development of a measurement system using computer vision and a far field microscope. In: McClung RC, Newman JC Jr (eds) Advances in Fatigue Crack Closure Measurement and Analysis: Second Volume, ASTM 1343. American Society for Testing and Materials, West Conshohocken, PA, pp 145156.

25. Riddell WT, Piascik RS, Sutton MA, Zhao W, McNeill SR, Helm JD (1999) Determining fatigue crack opening loads from near crack tip displacement measurements. In: McClung RC, Newman JC Jr (eds) Advances in Fatigue Crack Closure Measurement and Analysis: Second Volume, ASTM 1343. American Society for Testing and Materials, West Conshohocken, PA, pp 157174

26. Scrivens WA, Luo Y, Sutton MA, Collette SA, Myrick ML, Miney P, Colavita PE, Reynolds AP, Li XD (2007) Development of patterns for digital image correlation measurements at reduced length scales. Exp Mech 47:63 77 